\title{
A Cross-Sectional Study on the Correlation Between Inflammatory Cytokines, Negative Emotions, and Onset of Peripheral Neuropathy in Type 2 Diabetes
}

This article was published in the following Dove Press journal:

Neuropsychiatric Disease and Treatment

\author{
Ya-Hong Zheng ${ }^{1}, *$ \\ Chong-Yang Ren ${ }^{2, *}$ \\ Ying Shen ${ }^{3}$ \\ Jia-Bin $\mathrm{Li}^{1,4-6}$ \\ Ming-Wei Chen ${ }^{7}$
}

'Department of Infectious Diseases, The First Affiliated Hospital of Anhui Medical University, Hefei 230022, People's Republic of China; ${ }^{2}$ Department of Neurology (Sleep Disorder), The Affiliated Chaohu Hospital of Anhui Medical University, Chaohu 238000, People's Republic of China; ${ }^{3}$ Department of Endocrinology, Nanjing Tongren Hospital, Nanjing 2I I I02, People's Republic of China; ${ }^{4}$ Department of Infectious Diseases, The Affiliated Chaohu Hospital of Anhui Medical University, Chaohu 238000, People's Republic of China; ${ }^{5}$ Anhui Center for Surveillance of Bacterial Resistance, Hefei 230022. People's Republic of China; 'Institute of Bacterial Resistance, Anhui Medical University, Hefei 230022, People's Republic of China; ${ }^{7}$ Department of Endocrinology, The First Affiliated Hospital of Anhui Medical University, Hefei 230022, People's Republic of China

*These authors contributed equally to this work

Correspondence: Ming-Wei Chen Department of Endocrinology, The First Affiliated Hospital of Anhui Medical University, Hefei, People's Republic of China

Emailchmwl@I63.com

Jia-Bin $\mathrm{Li}$

Department of Infectious Diseases, The First Affiliated Hospital of Anhui Medical University, Hefei, People's Republic of

China

Email lijiabin@ahmu.edu.cn
Objective: This study explored the changes in the levels of IL-6, IL-17, TNF- $\alpha$, and TNF- $\beta$, whether such changes were associated with anxiety and depression in diabetic peripheral neuropathy (DPN), and what factors associated with the occurrence of DPN.

Methods: Forty-four patients diagnosed with DPN comprised the DPN group, including DPN1 (mild diabetic peripheral neuropathy, 29 cases) and DPN2 groups (moderate-severe diabetic peripheral neuropathy, 15 cases). Thirty-seven individuals with type 2 diabetes mellitus constituted the diabetes mellitus with no neuropathy (NDPN) group. Electromyography was applied to confirm DPN, and the Toronto clinical scoring system (TCSS) score was used to assess the severity of DPN. All subjects' emotions were evaluated using the self-rating anxiety scale (SAS) and self-rating depression scale (SDS). Triiodothyronine $\left(\mathrm{T}_{3}\right)$, tetraiodothyronine $\left(\mathrm{T}_{4}\right)$, and thyroid-stimulating hormone (TSH) levels were measured using chemiluminescent immunoassay. The relevant biochemical indicators were detected using an automatic biochemical analyzer. The plasma levels of cytokines were detected using quantitative sandwich enzyme-linked immunosorbent assay.

Results: Patients with DPN had elevated levels of anxiety, IL-6, IL-17, and TNF- $\alpha$. There were some positive associations between negative emotions and cytokines. The TCSS score positively correlated with IL-17, SAS score, and $\mathrm{T}_{3}$. DPN independently correlated with age, disease duration, fasting plasma glucose (FPG), and IL-17. The combination of IL-17 and TNF- $\alpha$ had higher diagnostic value for DPN than any single cytokine.

Conclusion: Patients with DPN had elevated levels of inflammatory cytokines, which were associated with negative emotion, and IL-17 had independent correlation with DPN.

Keywords: diabetic peripheral neuropathy, emotions, cytokines

\section{Introduction}

The high incidence of type 2 diabetes mellitus (T2DM) is a major global health problem, ${ }^{1}$ and its high mortality and commonly associated chronic complications are a major burden on human health, ${ }^{2}$ as well as an adverse socioeconomic factor. Diabetic peripheral neuropathy (DPN) is the most common chronic neurological complication of T2DM, ${ }^{3}$ with manifestations of sensory and motor nerve injury. It tends to occur after 50 years of age and is mostly found in patients with long-standing diabetes. ${ }^{4}$ Patients with DPN typically present with pain, numbness, tingling, weakness, and difficulties with balance, and less commonly with insomnia, anxiety, depression, and 
even suicidal thoughts. ${ }^{5}$ Moreover, DPN is a major risk factor for foot ulceration and amputation. ${ }^{6}$ Limited medicines and methods for treating DPN can eventually lead to complex medical care issues and have a serious influence on daily life. ${ }^{7}$

A recent study illustrated that the severity of chronic complications is related to the long-term accumulation of inflammation., 8 Several studies have also shown that patients with type 2 diabetic nephropathy (DN) exhibit high levels of diverse acute phase markers of inflammation, including C-reactive protein (CRP), serum amyloid A, fibrinogen, and IL-6. ${ }^{10,11}$ It is worthwhile to explore how inflammatory factor levels in patients with DPN change and whether they are related to the severity of DPN. The overexpression of pro-inflammatory cytokines represents a state of inflammation and stress in the body. Recent research has indicated that TNF- $\alpha$ and IL-6 play critical roles in the pathogenesis of DPN. ${ }^{12}$ In a study, the level of IL-17 was higher in patients with diabetes mellitus than in controls. However, differences in IL-17 levels between diabetic patients with and without DPN were not observed. ${ }^{13}$ Regarding the above points, in this study, we will explore the changes in IL-6, IL-17, TNF- $\alpha$, and TNF- $\beta$ in patients with DPN.

Prior studies have estimated that $28 \%$ of DN patients have depression and $35 \%$ have moderate-severe anxiety, and a more recent multi-center study reported that $59 \%$ of DN patients had either condition. ${ }^{14}$ Consequently, the degree of anxiety and depression in patients with diabetic peripheral neuropathy also deserves our attention. Recent studies have demonstrated that elevated levels of inflammatory markers were observed in patients with depression or anxiety. ${ }^{15,16}$ It is worthwhile to further study the correlation between the changes in cytokine levels and the degree of negative emotions in DPN patients.

In addition to the above two aspects, the current study will also explore whether the levels of inflammatory markers have value in the diagnosis of DPN and what factors independently associate with the occurrence of DPN, in order to achieve the early prevention, diagnosis, and treatment of DPN.

\section{Materials and Methods Subjects}

This was a cross-sectional study of differences in emotion and inflammatory cytokine levels between DPN and NDPN patients. A total of 81 patients (47 male and 34 female) with T2DM were included in the study, with an average age of $58.13 \pm 12.63$ years. All participants were patients in the Department of Endocrinology, the First Affiliated Hospital of Anhui Medical University. Patients' general information, such as sex, age, diabetes duration, weight, height, systolic blood pressure (SBP) and diastolic blood pressure (DBP), and body mass index (BMI), was collected after obtaining a detailed medical history and performing relevant examinations. According to the results of electromyography investigations, patients with T2DM were further divided into three groups: 37 patients with no peripheral neuropathy (NDPN group), 29 patients with mild peripheral neuropathy (DPN1 group), and 15 patients with moderate-severe peripheral neuropathy (DPN2 group). The DPN1 and DPN2 groups constituted the DPN group. The study was performed with the approval of ethics committee of the First Affiliated Hospital of Anhui Medical University (No.Quick-PJ2020 -08-13). The study was conducted in accordance with the Declaration of Helsinki, and all subjects provided consent before enrollment.

Inclusion criteria: The diagnosis of T2DM complies with the 1999 World Health Organization's diagnostic criteria for diabetes. Specific content: 1) symptoms of diabetes mellitus such as polydipsia, polyuria, polyphagia, weight loss, and random blood glucose level $\geq 11.1 \mathrm{mmol} /$ $\mathrm{L}$; 2) fasting plasma glucose (FPG) $\geq 7.0 \mathrm{mmol} / \mathrm{L}$; 3) 2-h blood glucose $\geq 11.1 \mathrm{mmol} / \mathrm{L}$ on oral glucose tolerance test; BMI, $18-33 \mathrm{~kg} / \mathrm{m}^{2}$; and glycated haemoglobin $\left(\mathrm{HbA}_{1 \mathrm{c}}\right), 5-12 \%$.

Exclusion criteria: Patients with type 1 diabetes, severe peripheral vascular disease, or diabetic foot were excluded. Patients with central or peripheral neuropathy (such as stroke, Guillain-Barre syndrome, spinal disorders), haematologic disorders, benign or malignant tumors, infections, autoimmune diseases, liver or kidney failure, or cardiac insufficiency were also excluded. In addition, individuals with mental illness, significant cognitive impairment leading to a lack of ability to complete the assessment, or who received psychotropic medications, vitamin $\mathrm{D}$, or nutritional medications were not included as subjects.

\section{Blood Sample Collection and Storage}

Blood samples were obtained from the median cubital vein in all subjects between 7 a.m. and 8 a.m. while quietly sitting after 12 hours of fasting and 30 minutes of rest. The blood samples were placed in blood collection tubes 
containing ethylene diamine tetra-acetic acid and sent to the biochemistry laboratory of the First Affiliated Hospital of Anhui Medical University. The serum was centrifuged at $4000 \mathrm{r} / \mathrm{min}, 25^{\circ} \mathrm{C}$ for $15 \mathrm{~min}$ and then placed in a $-80^{\circ} \mathrm{C}$ refrigerator prior to relevant measurements.

\section{Biochemical Analysis}

A chemiluminescence immunoassay system (Cobas 601 analyzer) was used to determine triiodothyronine $\left(\mathrm{T}_{3}\right)$, tetraiodothyronine $\left(\mathrm{T}_{4}\right)$, and thyroid-stimulating hormone (TSH) levels. A fully automatic biochemical analyzer (MODULE P800, Roche, Switzerland) was adopted to measure FPG, $\mathrm{HbA}_{1 \mathrm{c}}$, triglyceride (TG), total cholesterol (TC), high-density lipoprotein (HDL), low-density lipoprotein (LDL), total protein (TP), and albumin (ALB) levels. FPG was determined using the glucose oxidase method, TG and TC were determined using the enzyme method, and LDL and HDL were detected using the direct method.

\section{Enzyme-Linked Immunosorbent Assay (ELISA)}

ELISA was performed according to the instructions of the ELISA kits (Shanghai Yuanye Biological Technology Company). The concentrations of IL-6, IL-17, TNF- $\alpha$, and TNF- $\beta$ were calculated according to the standard curve.

\section{Psychological Status}

The self-rating depression scale (SDS) ${ }^{17}$ and self-rating anxiety scale (SAS) ${ }^{18}$ were designed to quantify the level of anxiety and depression in patients with anxiety or depression-related symptoms. The self-administered test has 20 questions, and each question is scored on a scale of 1 to 4 ( 1 = "a little of the time", 2 = "some of the time", 3 = "a good part of the time", 4 = "most of the time"). The doctor evaluated the scores after the 20 questions were answered by the patients.

\section{Electromyography}

American Nicolet Viking IV electromyography was applied to detect motor nerve conduction velocity (MCV) and subsensory nerve conduction velocity (SCV), including the median nerve, ulnar nerve, tibial nerve, and peroneal nerve, in patients with T2DM. ${ }^{19}$ The inspection was conducted by a professional technician and the temperature of the inspection room was controlled at $26-28^{\circ} \mathrm{C}$. DPN was diagnosed if there were two or more nerves with slower conduction velocity or longer conduction latency.

\section{Toronto Clinical Scoring System (TCSS)}

The TCSS score ${ }^{20}$ has been used to assess the severity of DPN and comprises three parts: peripheral symptom score (maximum of six points), deep tendon reflex score (maximum of eight points), and sensory test score (maximum of five points). The maximum score is 19 , and patients with more than 5 points were regarded as having clinical peripheral neuropathy. A total score of $0-5$ represents no neuropathy, $6-8$ represents mild neuropathy, 9-11 represents moderate neuropathy, and $12-19$ represents severe neuropathy.

\section{Statistical Analyses}

Normally distributed variables are presented as mean \pm standard deviation (SD). For non-parametric data, the variables are expressed as the 25th, 50th, and 75th percentiles [P50 (P25, P75)]. The chi-square test was used to analyze the differences between groups of categorical variables. One-way analysis of variance (ANOVA) and the least significant difference test were used for the overall and pairwise comparison between the three groups of normally distributed data, while the Kruskal-Wallis test and Kruskal-Wallis one-way ANOVA test (all pairwise) were used for nonnormally distributed variables. Non-parametric correlation analysis was adopted to test for correlations between the related blood indicators. Predicting the influence of various indicators on neuropathy was analyzed by multiple logistic regression. For the relationships existing among measures in the DPN group, a multivariate data reduction technique of principal component analysis was used. Receiver operating characteristics (ROC) analysis was performed to compare the diagnostic information provided by IL- 6 , IL-17, TNF- $\alpha$, and TNF- $\beta$, according to the area under the ROC curve (AUC $C_{R O C}$ ), optimal cut-off points, sensitivity, and specificity. The level of statistical significance was set at a twotailed P-value of 0.05 . The data obtained were all processed using SPSS 16.0 statistical software.

\section{Result}

Comparison of general information, and anxiety and depression assessment scores between the three groups

There was no statistical difference in sex, BMI, DBP, or SDS score among the three groups (see Table 1). There were statistically significant differences in age, disease duration, SBP, SAS scores, and HAMA scores. The age of the DPN1 $(\mathrm{P}=0.001)$ and DPN2 $(\mathrm{P}=0.007)$ groups was higher than that of the NDPN group. The patients in 
the DPN1 $(\mathrm{P}=0.001)$ and DPN2 $(\mathrm{P}=0.013)$ groups had a longer disease duration than the NDPN group. The SBP $(\mathrm{P}=0.008)$ and SAS scores $(\mathrm{P}=0.008)$ in the DPN2 group were higher than those in the NDPN group.

\section{Comparison of Various Indexes Between the Three Groups}

There was no statistically significant difference in $\mathrm{HbA}_{1 \mathrm{c}}$, $\mathrm{T}_{4}$, TSH, TP, ALB, TC, TG, or LDL among the three groups (see Table 1). Compared with that in the NDPN $(\mathrm{P}=0.004)$ and NPN1 $(\mathrm{P}=0.003)$ groups, the level of $\mathrm{T}_{3}$ was significantly higher in the DPN2 group. The patients in the DPN2 group also had a higher HDL than those in the NDPN group $(\mathrm{P}=0.012)$.

\section{Serum Cytokine Levels Between the Three Groups}

There were statistically significant differences in the levels of IL-6, IL-17, and TNF- $\alpha$ (see Table 1). In the pairwise comparison, a higher level of IL- 6 was observed in the
DPN1 $(P=0.039)$ and DPN2 $(P=0.023)$ groups than in the NDPN group. Compared with that in the NDPN group, the level of IL-17 was significantly higher in the DPN2 group $(\mathrm{P}=0.002)$. The patients in the DPN1 $(\mathrm{P}=0.021)$ and DPN2 $(\mathrm{P}=0.026)$ groups had a higher level of TNF- $\alpha$ than those in the NDPN group.

\section{Correlation Analysis Results in DPN Group}

As shown in Table 2, the SDS score was positively correlated with TNF- $\beta(\mathrm{r}=0.362, \mathrm{P}=0.016)$ in the DPN group, and the SAS score correlated with IL-6 $(\mathrm{r}=0.334, \mathrm{P}=$ $0.027)$, IL-17 ( $r=0.392, P=0.009)$, and TNF- $\beta$ ( $r=0.3$, $\mathrm{P}=0.048$ ) levels. After Bonferroni correction, no significant was found in the correlation between negative emotion and biochemical indicators.

Spearman correlation analysis showed negative associations between the level of TSH and IL-6 $(\mathrm{r}=-0.320$, $\mathrm{P}=0.034)$ and TNF- $\alpha(\mathrm{r}=-0.338, \mathrm{P}=0.025)$. The level of $\mathrm{T}_{3}$ was positively correlated with IL-17 $(\mathrm{r}=0.419, \mathrm{P}=$

Table I The Comparison of General Data, Emotional Assessment, Biochemical Indicators and Cytokines Among Three Groups

\begin{tabular}{|c|c|c|c|c|c|}
\hline Variables & $\operatorname{NDPN}(n=37)$ & DPN-I $(n=29)$ & DPN-2 $(n=15)$ & Statistic & p-values \\
\hline Male/Female & $23 / 14$ & $17 / 12$ & $7 / 8$ & 1.059 & 0.589 \\
\hline Age $(y r)$ & $49.3 \pm 14.2$ & $60.5 \pm 10.8^{*}$ & $60.0 \pm 10.6^{*}$ & -7.843 & 0.001 \\
\hline Disease duration (yr) & $9.0(6.0,12.0)$ & $12.0(5.5,16.5)^{*}$ & $10.0(6.0,17.0)^{*}$ & 15.918 & $<0.001$ \\
\hline BMI $(\mathrm{kg} / \mathrm{m} 2)$ & $24.3 \pm 3.7$ & $25.9(24.6,27.3)$ & $25.1 \pm 3.9$ & 3.490 & 0.175 \\
\hline $\mathrm{SBP}(\mathrm{mmHg})$ & $129.8 \pm 19.1$ & $136.7 \pm 13.0$ & $146.0(\mid 37.0,160.0)^{*}$ & 9.909 & 0.007 \\
\hline $\mathrm{DBP}(\mathrm{mmHg})$ & $81.4 \pm 14.4$ & $81.0(69.5 .0,87.0)$ & $83.9 \pm 11.9$ & 0.833 & 0.659 \\
\hline SDS (score) & $55.3 \pm 9.4$ & $60.5 \pm 9.8$ & $59.9 \pm 8.1$ & 2.885 & 0.062 \\
\hline SAS (score) & $47.7 \pm 7.9$ & $51.0(46.0,56.0)$ & $56.2 \pm 9.7^{* \#}$ & 9.611 & 0.008 \\
\hline FPG $(\mathrm{mmol} / \mathrm{l})$ & $7.8(6.2,10.3)$ & $9.8 \pm 2.9$ & $9.6 \pm 2.1$ & 5.448 & 0.066 \\
\hline $\mathrm{HbA}_{\mathrm{Ic}}(\%)$ & $9.0(7.9,10.5)$ & $9.0(7.6,10.7)$ & $9.8 \pm 2.1$ & 0.910 & 0.634 \\
\hline $\mathrm{T}_{3}(\mathrm{pmol} / \mathrm{l})$ & $1.7(1.5,1.9)$ & $1.7(1.5,1.9)$ & $2.0 \pm 0.3^{* \#}$ & 10.124 & 0.006 \\
\hline $\mathrm{T}_{4}(\mathrm{pmol} / \mathrm{l})$ & $96.7(89.3,119.2)$ & $95.9(90.4,107.8)$ & $106.9 \pm 20.8$ & 0.778 & 0.678 \\
\hline TSH $(\mu \mathrm{IU} / \mathrm{mL})$ & $2.3(1.6,3.3)$ & $2.4 \pm 1.2$ & $2.5 \pm 1.4$ & 0.041 & 0.980 \\
\hline Total protein $(g / l)$ & $68.9 \pm 5.3$ & $70.1 \pm 6.4$ & $66.0 \pm 6.9$ & 2.302 & 0.107 \\
\hline Albumin $(g / l)$ & $42.6(40.8,46.5)$ & $42.4 \pm 5.4$ & $40.5 \pm 4.6$ & 3.514 & 0.173 \\
\hline Total cholesterol $(\mathrm{mmol} / \mathrm{l})$ & $4.9 \pm 1.3$ & $4.6 \pm 1.2$ & $5.1 \pm 0.7$ & 0.978 & 0.381 \\
\hline Triglyceride $(\mathrm{mmol} / \mathrm{l})$ & $1.7(1.2,3.1)$ & $1.6(1.0,2.2)$ & I.2(0.9, 2.6) & 2.537 & 0.281 \\
\hline LDL (mmol/l) & $2.9 \pm 1.0$ & $2.3 \pm 1.2$ & $3.0(2.8,3.3)$ & 4.070 & 0.131 \\
\hline $\mathrm{HDL}(\mathrm{mmol} / \mathrm{l})$ & I.I $(0.9, \mathrm{I} .5)$ & $1.3 \pm 0.4$ & $1.5 \pm 0.4^{*}$ & 8.356 & 0.015 \\
\hline Interleukin-6 (pg/mL) & II.I(7.0, 40.3) & $41.5(8.3,60.3)^{*}$ & $48.1(10.6,59.8)^{*}$ & 6.993 & 0.030 \\
\hline Interleukin-I7 (pg/mL) & $8.3(6.5,46.0)$ & $26.9(6.8,64.9)$ & $60.4(17.7 .6,64.9)^{*}$ & $|1.54|$ & 0.003 \\
\hline TNF- $\alpha(\mathrm{pg} / \mathrm{mL})$ & $35.7(5.0,83.5)$ & $85.6(29.2$ 98.9)* & $76.0(23.0,103.2)^{*}$ & 7.602 & 0.022 \\
\hline TNF- $\beta$ (pg/mL) & $34.0(4.4,50.8)$ & $53.9(15.3,62.7)$ & $44.9(26.9,60.9)$ & 4,845 & 0.089 \\
\hline
\end{tabular}

Notes: Compared with NDPN group, *P < 0.05; Compared with DPNI group, ${ }^{*} \mathrm{P}<0.05$

Abbreviations: BMI, body mass index; DBP, diastolic blood pressure; DPN-I, mild diabetic peripheral neuropathy; DPN-2, moderate-severe diabetic peripheral neuropathy; FPG, fasting plasma glucose; HbA $\mathrm{Ic}_{\mathrm{c}}$, glycosylated hemoglobin Al c; HDL, high-density lipoprotein cholesterol; LDL, low-density lipoprotein cholesterol; NDPN, no diabetic peripheral neuropathy; SAS, self-rating anxiety scale; SBP, systolic blood pressure; SDS, self-rating depression scale; $\mathrm{T}_{3}$, triiodothyronine; $\mathrm{T}_{4}$, tetraiodothyronine; TNF- $\alpha$, tumor necrosis factor- $\alpha$; TNF- $\beta$, tumor necrosis factor- $\beta$; TSH, thyroid stimulating hormone. 
Table 2 The Correlations Between Negative Emotion and Biochemical Indicators

\begin{tabular}{|l|l|l|l|l|l|l|l|}
\hline Variables & IL-6 & IL-17 & TNF- $\alpha$ & TNF- $\beta$ & T $_{\mathbf{3}}$ & T $_{\mathbf{4}}$ & TSH \\
\hline SDS & 0.197 & -0.058 & 0.182 & $0.362^{*}$ & -0.075 & -0.074 & -0.139 \\
SAS & $0.334^{*}$ & $0.392^{*}$ & 0.119 & $0.300^{*}$ & -0.139 & -0.095 & -0.054 \\
\hline
\end{tabular}

Note: ${ }^{*}$ Correlation is significant at the 0.05 level (2-tailed).

Abbreviations: IL-6, interleukin-6; IL- I7, interleukin- I7; SAS, self-rating anxiety scale; SDS, self-rating depression scale; $\mathrm{T}_{3}$, triiodothyronine; $\mathrm{T}_{4}$, tetraiodothyronine; TNF$\alpha$, tumor necrosis factor- $\alpha$; TNF- $\beta$, tumor necrosis factor- $\beta$; TSH, thyroid stimulating hormone.

Table 3 Logistic Regression Analysis on Risk Factors of DPN

\begin{tabular}{|l|l|l|l|l|l|l|}
\hline Variables & $\boldsymbol{\beta}$ & SE & Wald $\mathbf{X}^{\mathbf{2}}$ & P value & OR & $\mathbf{9 5 \%}$ Cl \\
\hline Age $(\mathrm{yr})$ & 0.075 & 0.03 & 6.34 & 0.012 & 1.078 & $1.017-1.142$ \\
Disease duration $(\mathrm{yr})$ & 0.120 & 0.048 & 6.245 & 0.012 & 1.127 & $1.026-1.238$ \\
FPG (mmol/l) & 0.384 & 0.132 & 8.485 & 0.004 & 1.469 & $1.134-1.902$ \\
IL-I7 (pg/mL) & 0.037 & 0.013 & 7.514 & 0.006 & 1.037 & $1.010-1.065$ \\
Constant & -9.647 & 2.606 & 13.707 & 0.000 & 0.000 & \\
\hline
\end{tabular}

Abbreviations: $95 \% \mathrm{Cl}$, 95\% confidence interval; FPG, fasting plasma glucose; IL-17, interleukin- I7; OR, odds ratio; SE, standard error; $\beta$, coefficients.

0.005 ). The TCSS score positively correlated with IL-17 ( $\mathrm{r}$ $=0.309, \mathrm{P}=0.041)$, SAS score $(\mathrm{r}=0.321, \mathrm{P}=0.034)$, and $\mathrm{T}_{3}(\mathrm{r}=0.364, \mathrm{P}=0.0115)$. After Bonferroni correction, significant was only found in the correlation between $\mathrm{T} 3$ and IL-17 $(r=0.419, P=0.005)$.

\section{Logistic Regression Analysis}

Logistic regression analysis was conducted with the presence/absence of DPN as the dependent variable and age, disease duration, BMI, FPG, IL-6, IL-17, TNF- $\alpha$, TNF- $\beta$, and HDL as independent variables in the patient group. The results showed that DPN was independently correlated with age, disease duration, FPG, and IL-17 (Table 3).

\section{Principal Component Analysis for DPN}

Principal components analysis was employed in this study to avoid the interference of confounding factors for correlations between different variables. The results can reflect the correlation of variables after the elimination of mutual interference factors. The Kaiser-Meyer-Olkin (KMO) was $0.550(>0.500)$ and $\mathrm{P}<0.001$ in Bartlett's test of sphericity in the DPN group, suggesting that the sample size is suitable and the indexes selected are appropriate for principle components analysis. TCSS score, disease duration, BMI, FPG, HbA 1 , IL-6, IL-17, TNF- $\alpha$, TNF- $\beta, T_{3}, T_{4}$, $\mathrm{TSH}$, SDS score, and SAS score were included. Five significant factors were identified, and the component loading of variables on the rotated factors are shown in Table 4. The four cytokines loaded highly on factor 1 (absolute value of coefficient $>0.5$ ). Disease duration,
BMI, $\mathrm{T}_{3}$, and $\mathrm{T}_{4}$ loaded highly on factor 2 . FPG, $\mathrm{HbA}_{1 \mathrm{c}}$, and SDS score loaded highly on factor 3. TCSS and SAS scores loaded highly on factor 4 . BMI and TSH loaded highly on factor 5 .

\section{ROC Curve for Serum Cytokines Levels}

ROC curve analysis was performed on the cytokines levels in 81 subjects (including DPN and NDPN) to determine whether those levels had diagnostic value for DPN. The $\mathrm{AUC}_{\mathrm{ROC}}$ for each cytokine was detected at the level less than $70.0 \%$ sensitivity and specificity. Regarding a combined diagnostic method (only for two cytokines), a combination of IL-17 and TNF- $\alpha$ exhibited a diagnostic value more than $70.0 \%\left(\mathrm{AUC}_{\mathrm{ROC}}=70.8 \%\right)$ for $\mathrm{DPN}$; the sensitivity and specificity were $62.2 \%$ and $72.7 \%$, respectively (see Table 5 and Figure 1).

\section{Discussion}

The current results show that the expression levels of IL-6, IL-17, and TNF- $\alpha$ in the DPN1, DPN2, and NDPN groups were different. In brief, subjects with varying degrees of DPN had higher levels of the three cytokines, demonstrating that DPN extended the chronic low-grade inflammatory state seen in T2DM. In further support of this view, the results of the principal component analysis (avoiding the interference of confounding factors) demonstrated that these four cytokines loaded highly on factor 1 and were positively linked. A similar study reported elevated CRP, IL-6, and IL-18 in patients with DN. ${ }^{21,22}$ A study of 32 patients with T2DM showed that increased serum IL-6 
Table 4 Principal Component Analysis for DPN Group

\begin{tabular}{|c|c|c|c|c|c|c|}
\hline Terms & Measures & Factor I & Factor 2 & Factor 3 & Factor 4 & Factor 5 \\
\hline Severity of disease & TCSS & $0.17 \mid$ & 0.410 & -0.061 & $0.769 *$ & 0.076 \\
\hline Duration of disease & Duration & 0.063 & $-0.554 *$ & -0.037 & 0.296 & -0.113 \\
\hline General data & Body mass index & -0.193 & $-0.582 *$ & 0.110 & 0.213 & $0.520 *$ \\
\hline Blood glucose & $\begin{array}{l}\text { Fasting plasma glucose } \\
\text { Glycosylated hemoglobin Alc }\end{array}$ & $\begin{array}{l}-0.033 \\
0.052\end{array}$ & $\begin{array}{l}0.040 \\
0.375\end{array}$ & $\begin{array}{l}0.890 * \\
0.809 *\end{array}$ & $\begin{array}{l}0.127 \\
0.222\end{array}$ & $\begin{array}{l}0.041 \\
-0.042\end{array}$ \\
\hline Cytokines & $\begin{array}{l}\text { Interleukin-6 } \\
\text { Interleukin- I7 } \\
\text { Tumor necrosis factor- } \alpha \\
\text { Tumor necrosis factor- } \beta\end{array}$ & $\begin{array}{l}0.887^{*} \\
0.812^{*} \\
0.810^{*} \\
0.841^{*}\end{array}$ & $\begin{array}{l}-0.086 \\
0.138 \\
-0.181 \\
-0.243\end{array}$ & $\begin{array}{l}-0.142 \\
-0.267 \\
0.140 \\
0.109\end{array}$ & $\begin{array}{l}-0.080 \\
0.211 \\
-0.253 \\
-0.219\end{array}$ & $\begin{array}{l}0.021 \\
0.023 \\
0.222 \\
0.198\end{array}$ \\
\hline Thyroid hormones & $\begin{array}{l}\text { Triiodothyronine } \\
\text { Tetraiodothyronine } \\
\text { Thyroid stimulating hormone }\end{array}$ & $\begin{array}{l}0.443 \\
0.065 \\
-0.464\end{array}$ & $\begin{array}{l}0.628^{*} \\
0.729 * \\
-0.018\end{array}$ & $\begin{array}{l}-0.114 \\
0.089 \\
-0.188\end{array}$ & $\begin{array}{l}0.026 \\
-0.279 \\
0.160\end{array}$ & $\begin{array}{l}0.164 \\
0.303 \\
0.743^{*}\end{array}$ \\
\hline Emotional assessment & $\begin{array}{l}\text { Self-rating depression scale } \\
\text { Self-rating anxiety scale }\end{array}$ & $\begin{array}{l}0.306 \\
0.422\end{array}$ & $\begin{array}{l}-0.407 \\
-0.140\end{array}$ & $\begin{array}{l}0.50 I^{*} \\
-0.089\end{array}$ & $\begin{array}{l}-0.075 \\
0.656 *\end{array}$ & $\begin{array}{l}0.038 \\
-0.108\end{array}$ \\
\hline $\begin{array}{l}\text { Eigenvalues } \\
\text { Variance accounted for (\%) }\end{array}$ & & $\begin{array}{l}3.569 \\
25.496\end{array}$ & $\begin{array}{l}2.185 \\
15.609\end{array}$ & $\begin{array}{l}1.903 \\
13.591\end{array}$ & $\begin{array}{l}1.492 \\
10.658\end{array}$ & $\begin{array}{l}1.066 \\
7.614\end{array}$ \\
\hline
\end{tabular}

Note: *Loadings higher than 0.5 .

Table 5 AUCROC Curve for Serum TNF- $\alpha$, TNF- $\beta$, IL-6 and IL- I7 Level

\begin{tabular}{|l|l|l|l|l|l|}
\hline Variables & IL-6 & IL-I 7 & TNF- $\boldsymbol{\alpha}$ & TNF- $\boldsymbol{\beta}$ & IL-I 7+TNF- $\boldsymbol{\alpha}$ \\
\hline Area & 0.677 & 0.679 & 0.677 & 0.643 & 0.706 \\
$95 \% \mathrm{Cl}$ & $0.549-0.785$ & $0.563-0.795$ & $0.559-0.795$ & $0.520-0.765$ & $0.592-0.820$ \\
Threshold value & 37.4753 & 59.2184 & 85.0563 & $54.25 \mathrm{I}$ & - \\
Sensitivity & 0.568 & 0.386 & 0.5 & 0.477 & 0.622 \\
Specificity & 0.757 & 0.946 & 0.811 & 0.811 & 0.727 \\
Yoden index & 0.325 & 0.332 & 0.311 & 0.288 & 0.349 \\
\hline
\end{tabular}

Abbreviations: $95 \% \mathrm{CI}$, 95\% confidence interval; IL-6, interleukin-6; IL-I7, interleukin- I7; TNF- $\alpha$, tumor necrosis factor- $\alpha$; TNF- $\beta$, tumor necrosis factor- $\beta$.

levels were associated with markers of sensory and motor axonal damage to large nerve fibres, suggesting that IL-6 may play a role in the pathogenesis of nerve fibre injury. ${ }^{23}$ Therefore, the negative stimulatory effect of high inflammation on peripheral nerves or inhibition of neuroprotective mechanisms by diabetes may be related to the pathogenesis of peripheral neuropathy.

The current study initially found that the expression of serum IL-17 in DPN2 patients was higher than that in patients in the NDPN group, inconsistent with previous research. ${ }^{13}$ The differences in enrolment criteria, sample size, region, and population, as well as the differences in the severity of DPN at the time of hospitalization, may led to the inconsistency of research results. IL-17 has an inflammatory effect, but it also plays some roles in homeostasis, nerve protection, and tissue repair. ${ }^{24}$ IL-17 was over-expressed in patients with diabetes, but low expression of IL-17 was detected in the nerves of diabetic mice. ${ }^{25}$ There is no reasonable explanation yet, but some aspects deserve our attention. First, in the current study, the increase in inflammatory cytokines were in $\mathrm{pg} / \mathrm{mL}$, but an injection of $10 \mathrm{ng} / \mathrm{mL}$ (minimum) of IL-17A promoted nerve growth in diabetic mice. ${ }^{25}$ Based on this, we questioned whether systemic inflammation is related to the expression of inflammatory cytokines in the area of neuropathy and whether the effect of IL-17 is concentration-dependent. Second, it is noteworthy that the IL-17 level was reduced only in the sciatic nerve of DM mice, and no other peripheral nerve studies were conducted. 


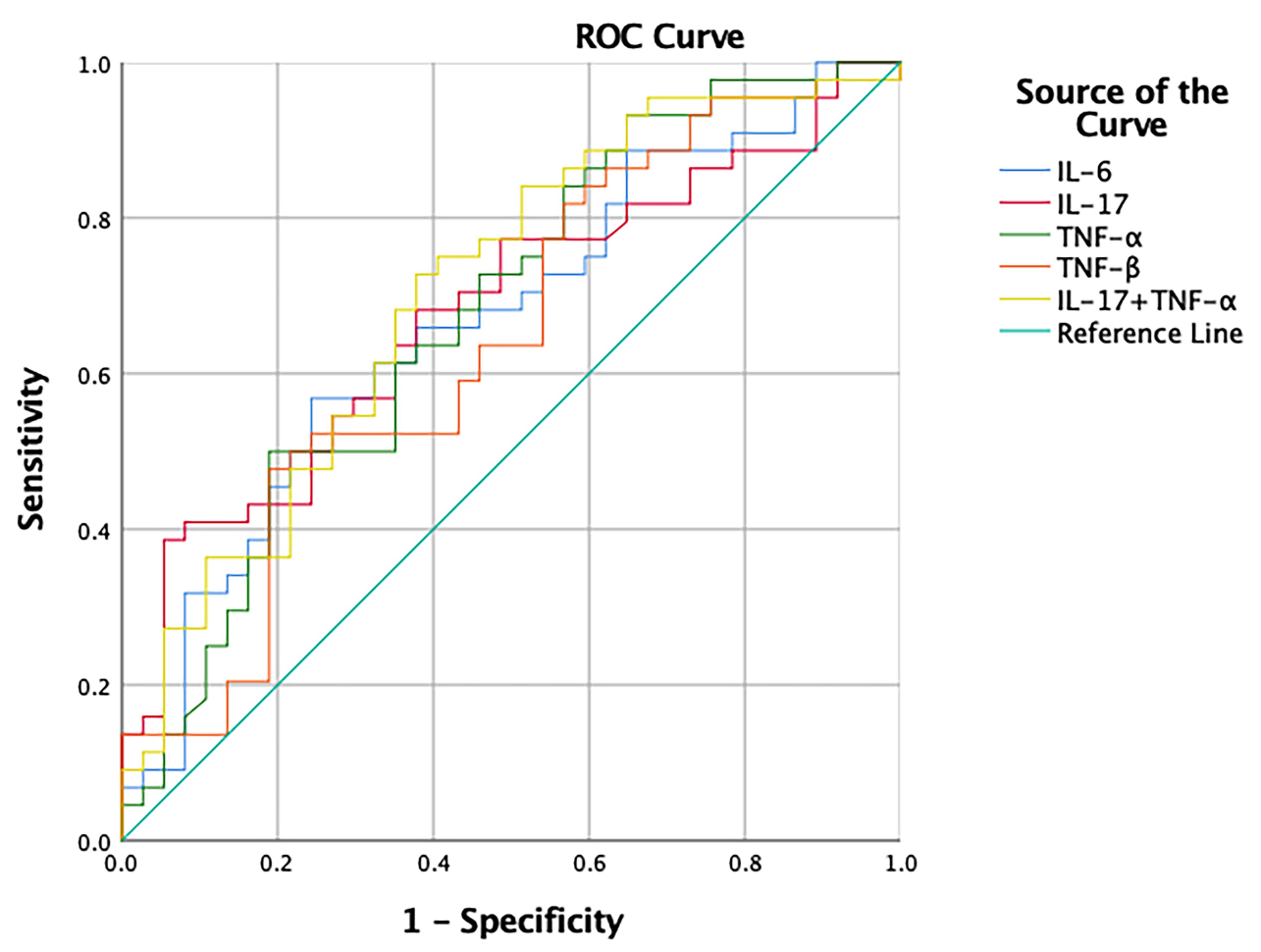

Figure I Receiver operating characteristics (ROC) curve for serum cytokines levels.

This study found that the SAS scores in the DPN2 group were higher than those in the NDPN group, suggesting that patients with moderate-severe DPN were in a state of anxiety. It is worth mentioning that although there was no statistically significant difference, patients with mild and moderatesevere DPN had a certain degree of depression. These results are consistent with those of previous studies. For instance, a recent review showed that patients with depression were more likely to suffer from T2DM and DPN. ${ }^{26}$ Early prevention and symptomatic treatment of depression can reverse this risk factor for DPN. ${ }^{27}$ Anxiety disorders also increase the risk of DPN, and the neurobiological mechanisms affecting this comorbidity have been studied in rat models. ${ }^{28}$ However, the inclusion of relevant variables in the current study did not reveal that anxiety and depression were independent risk factors for DPN. This may be because the sample size of this study was small as it was not a large-sample epidemiological survey. Additionally, although the levels of anxiety and depression in the DPN group were higher than those in the NDPN group, these negative emotions were not enough to affect the incidence of DPN.

In addition, the SAS score positively correlated with IL-6, IL-17, and TNF- $\beta$ levels in the DPN group. Unfortunately, these correlations were not as significant after Bonferroni correction, which was the limitation of this study. We believe that a larger sample size is needed to support this correlation. Previous studies have shown elevated serum IL-17 levels in patients with rheumatoid arthritis associated with anxiety, and the IL-17 level was independently associated with higher anxiety scores, which is consistent with our results. ${ }^{29}$ Similar results have been reported that IL- 6 and IFN- $\gamma$ levels positively correlated with self-assessed anxiety and depressive symptoms. ${ }^{30}$ However, these studies all have some limitations. First, the inclusion criteria of the subjects were not strict enough. Second, in these studies, there were errors caused by the interference of physical diseases. In addition, the patients' medication histories during the experiment were inconsistent. Despite this, there is some evidence of the positive relationship between inflammation and negative emotion. People with allergies are more likely to suffer from anxiety and depression than untreated people, ${ }^{31}$ supporting the hypothesis that the mediators of allergic inflammation may directly influence the centers of the brain involved in emotions. ${ }^{32}$

However, there is a lack of research on the correlation between DPN severity, inflammatory factors, and emotional status. The current research has carried out analyses of this; the results show that for DPN patients, the TCSS score positively correlated with IL-17 levels and anxiety 
scores. Although these correlations were not significant after Bonferroni correction, the results of the principal component analysis also demonstrated that the TCSS score and anxiety score positively correlated with high load in factor 4. In short, DPN patients with more severe neuropathy showed higher levels of IL-17 and more anxiety. The TCSS is an effective screening tool for DPN and has a higher diagnostic value for DPN than the Michigan Neuropathy Screening Scoring System, making it appropriate for the clinical diagnosis of DPN and analysis of the severity of the disease. ${ }^{33}$ Our results showed that only IL17 was related to DPN, indicating that IL-17 is more closely related to the occurrence of DPN than other cytokines.

The results of the logistic regression analysis show that age, course of disease, FPG, and IL-17 were independent risk factors related to the development of DPN. T2DM patients with advanced age, long course of disease, high level of FPG, and increased expression of IL-17 were more likely to develop symptoms of peripheral neuropathy, consistent with previous research. DPN was prevalent in overweight or obese T2DM patients in Guangdong province in China and was significantly associated with age, $\mathrm{HbA}_{1 \mathrm{c}}$, and duration of diabetes. ${ }^{34}$ Unlike in previous studies, the upregulation of cytokines, especially IL-17, may be a risk factor for DPN, which further supports the view that IL-17 is more closely related to the occurrence of DPN than are other cytokines.

In the current study, compared with each cytokine, a combination of IL-17 and TNF- $\alpha$ exhibited higher diagnostic value for DPN $\left(\mathrm{AUC}_{\mathrm{ROC}}=70.8 \%\right)$. The most prominent manifestation of DPN is a decrease of nerve conduction velocity or amplitude. Electromyography is mainly used for the diagnosis of DPN but there are some disadvantages. It is only possible to examine large myelinated fibres with limited inspection scope, and the test results of different hospitals and technicians have certain deviations. Furthermore, for diabetic patients, peripheral neuropathy is insidious in onset, mild in symptoms, and prone to misdiagnosis. Since the $\mathrm{AUC}_{\mathrm{ROC}}$ of each cytokine was more than $50 \%$ but less than $70 \%$, we believe that their diagnostic value for DPN was not sufficient. Therefore, two cytokines were selected for combined detection (the fewer the indicators, the more convenient and lower the cost for patients). It is believed that the combination of IL-17 and TNF- $\alpha$ has value in the diagnosis of DPN, at least as an auxiliary diagnostic method. In summary, the monitoring of cytokines may be helpful for timely detection of the risk of peripheral neuropathy.

This study also showed a link between thyroid hormones and cytokines in DPN patients. In the DPN group, TSH was negatively correlated with IL- 6 and TNF- $\alpha$; $T_{3}$ was positively correlated with IL-17. This suggests that changes in thyroid hormones (normal range) may be parallel to changes in cytokines. First, changes in immune function in DPN patients may affect the secretion of thyroid hormones and thyrotropin. Pro-inflammatory activities, such as increased TNF- $\alpha$ expression, may result from increased $\mathrm{T}_{3}$ levels. ${ }^{35}$ Second, the increased concentration of pro-inflammatory factors was accompanied by anxiety and depression, which are also related to thyroid dysfunction. For instance, patients with overt hyperthyroidism are frequently reported to have anxiety, fatigue, poor concentration, and disturbed sleep, all of which constitute core symptoms of generalized anxiety disorder. ${ }^{36}$ However, there was no direct correlation between negative emotions and thyroid hormones in this study, which may be related to the small sample size and inclusion criteria (patients with thyroid dysfunction were excluded from the current study to avoid confounding factors).

In the DPN group, the TCSS score positively correlated with $\mathrm{T}_{3}(\mathrm{r}=0.364, \mathrm{P}=0.015)$. It can be considered that the severity of DPN represented by the TCSS score has a parallel relationship with the $T_{3}$ levels. There are two interpretations. One is that the severity of DPN affects the $\mathrm{T}_{3}$ level. The other is that the increase in IL-17 caused by DPN affects the $T_{3}$ level.

\section{Conclusion}

In summary, DPN patients had elevated levels of inflammatory cytokines, which were associated with negative emotions, and IL-17 had independent correlation with DPN. The results of ROC curve proved that the combined monitoring of cytokine levels was helpful for the diagnosis of DPN. Cytokine detection and mood assessment should be paid more attention in the diagnosis and treatment of DPN. Effective interventions in these two areas may contribute to the remission of DPN. However, the current study has some deficiencies and limitations. First, the small sample size of the moderate-severe DPN group may have influenced some of the results. We can explore more relationships by expanding the sample size of the moderate-severe DPN group, as well as dividing it into separate moderate and severe groups. Second, this experiment was a preliminary cross-sectional study, so a cause 
and effect relationship between DPN and related parameters cannot be established. It is necessary for us to further explore inflammatory cytokines and negative emotions in DPN patients.

\section{Acknowledgments}

This study was supported by grants from the Key Research and Development Program of Anhui Province (grant numbers, 912096047147). The authors would like to thank the support and assistance from The Department of Endocrinology of The First Affiliated Hospital of Anhui Medical University. We thank the associate editor and the reviewers for their useful feedback that improved this paper.

\section{Disclosure}

The authors declare no conflicts of interest. All patients were informed about the purpose of the study.

\section{References}

1. Zhou J, Zhou S. Inflammation: therapeutic targets for diabetic neuropathy. Mol Neurobiol. 2014;49(1):536-546. doi:10.1007/ s12035-013-8537-0

2. King GL. The role of inflammatory cytokines in diabetes and its complications. J Periodontol. 2008;79(8 Suppl):1527-1534. doi:10.1902/jop.2008.080246

3. Tesfaye S, Boulton AJM, Dickenson AH. Mechanisms and management of diabetic painful distal symmetrical polyneuropathy. Diabetes Care. 2013;36(9):2456-2465. doi:10.2337/dc12-1964

4. Said G. Diabetic neuropathy-a review. Nat Clin Pract Neurol. 2007;3 (6):331-340. doi:10.1038/ncpneuro0504

5. Gore M, Brandenburg NA, Dukes E, Hoffman DL, Tai KS, Stacey B. Pain severity in diabetic peripheral neuropathy is associated with patient functioning, symptom levels of anxiety and depression, and sleep. J Pain Symptom Manage. 2005;30(4):374-385. doi:10.1016/j. jpainsymman.2005.04.009

6. Hussain G, Rizvi SA, Singhal S, Zubair M, Ahmad J. Cross sectional study to evaluate the effect of duration of type 2 diabetes mellitus on the nerve conduction velocity in diabetic peripheral neuropathy. Diabetes Metab Syndr. 2014;8(1):48-52. doi:10.1016/j. dsx.2013.02.003

7. Gok Metin Z, Arslan IE. Diabetic Peripheral Neuropathic Pain From the Perspective of Turkish Patients: A Qualitative Study. J Transcult Nurs. 2018;29(6):514-522. doi:10.1177/1043659617753044

8. Adamis AP. Is diabetic retinopathy an inflammatory disease? $\mathrm{Br}$ J Ophthalmol. 2002;86(4):363-365. doi:10.1136/bjo.86.4.363

9. Dalla Vestra M, Mussap M, Gallina P, et al. Acute-phase markers of inflammation and glomerular structure in patients with type 2 diabetes. $J$ Am Soc Nephrol. 2005;16(Suppl 1):S78-S82. doi:10.1681/asn.2004110961

10. Chow FY, Nikolic-Paterson DJ, Ozols E, Atkins RC, Tesch GH. Intercellular adhesion molecule-1 deficiency is protective against nephropathy in type 2 diabetic $\mathrm{db} / \mathrm{db}$ mice. $J$ Am Soc Nephrol. 2005;16(6):1711-1722. doi:10.1681/asn.2004070612

11. Hasegawa G, Nakano K, Sawada M, et al. Possible role of tumor necrosis factor and interleukin-1 in the development of diabetic nephropathy. Kidney Int. 1991;40(6):1007-1012. doi:10.1038/ ki.1991.308
12. Jin HY, Park TS. Role of inflammatory biomarkers in diabetic peripheral neuropathy. J Diabetes Investig. 2018;9(5):1016-1018. doi:10.1111/jdi.12794

13. Bilir B, Tulubas F, Bilir BE, et al. The association of vitamin D with inflammatory cytokines in diabetic peripheral neuropathy. $J$ Phys Ther Sci. 2016;28(7):2159-2163. doi:10.1589/jpts.28.2159

14. Boulanger L, Zhao Y, Bao Y, Russell MW. A retrospective study on the impact of comorbid depression or anxiety on healthcare resource use and costs among diabetic neuropathy patients. BMC Health Serv Res. 2009;9(1):111. doi:10.1186/1472-6963-9-111

15. Ur E, White PD, Grossman A. Hypothesis: cytokines may be activated to cause depressive illness and chronic fatigue syndrome. Eur Arch Psychiatry Clin Neurosci. 1992;241(5):317-322. doi:10.1007/ bf02195983

16. Maes M. Evidence for an immune response in major depression: a review and hypothesis. Prog Neuropsychopharmacol Biol Psychiatry. 1995;19(1):11-38. doi:10.1016/0278-5846(94)00101-m

17. Zung WW. A SELF-RATING DEPRESSION SCALE. Arch Gen Psychiatry. 1965;12:63-70. doi:10.1001/ archpsyc.1965.01720310065008

18. Jegede RO. Psychometric characteristics of Yoruba versions of Zung's self-rating depression scale and self-rating anxiety scale. Afr J Med Med Sci. 1979;8(3-4):133-137.

19. Ge S, Xie J, Zheng L, et al. Associations of serum anti-ganglioside antibodies and inflammatory markers in diabetic peripheral neuropathy. Diabetes Res Clin Pract. 2016;115:68-75. doi:10.1016/ j.diabres.2016.02.005

20. Perkins BA, Olaleye D, Zinman B, Bril V. Simple screening tests for peripheral neuropathy in the diabetes clinic. Diabetes Care. 2001;24 (2):250-256. doi:10.2337/diacare.24.2.250

21. Herder C, Lankisch M, Ziegler D, et al. Subclinical inflammation and diabetic polyneuropathy: MONICA/KORA Survey F3 (Augsburg, Germany). Diabetes Care. 2009;32(4):680-682. doi:10.2337/dc08-2011

22. Herder C, Bongaerts BW, Rathmann W, et al. Association of subclinical inflammation with polyneuropathy in the older population: KORA F4 study. Diabetes Care. 2013;36(11):3663-3670. doi: $10.2337 /$ dc13-0382

23. Magrinelli F, Briani C, Romano M, et al. The Association between Serum Cytokines and Damage to Large and Small Nerve Fibers in Diabetic Peripheral Neuropathy. J Diabetes Res. 2015;2015:547834. doi:10.1155/2015/547834

24. Littman DR, Rudensky AY. Th17 and regulatory T cells in mediating and restraining inflammation. Cell. 2010;140(6):845-858. doi:10.1016/j.cell.2010.02.021

25. Habash T, Saleh A, Roy Chowdhury SK, Smith DR, Fernyhough P. The proinflammatory cytokine, interleukin-17A, augments mitochondrial function and neurite outgrowth of cultured adult sensory neurons derived from normal and diabetic rats. Exp Neurol. 2015;273:177-189. doi:10.1016/j.expneurol.2015.08.016

26. Vas PRJ, Papanas N. Depression and Diabetic Peripheral Neuropathy: birds of a Feather, But When do They Flock Together? Exp Clin Endocrinol Diabetes. 2020;128(5):347-349. doi:10.1055/a-0808-4269

27. Yoo M, Sharma N, Pasnoor M, Kluding PM. Painful Diabetic Peripheral Neuropathy: presentations, Mechanisms, and Exercise Therapy. J Diabetes Metab. 2013;Suppl 10. doi:10.4172/2155-6156. S10-005

28. Smith KJ, Béland M, Clyde M, et al. Association of diabetes with anxiety: a systematic review and meta-analysis. J Psychosom Res. 2013;74(2):89-99. doi:10.1016/j.jpsychores.2012.11.013

29. Liu Y, Ho RC-M, Mak A. The role of interleukin (IL)-17 in anxiety and depression of patients with rheumatoid arthritis. Int J Rheum Dis. 2012;15(2):183-187. doi:10.1111/j.1756-185X.2011.01673.x

30. Henje Blom E, Lekander M, Ingvar M, Åsberg M, Mobarrez F, Serlachius E. Pro-inflammatory cytokines are elevated in adolescent females with emotional disorders not treated with SSRIs. J Affect Disord. 2012;136(3):716-723. doi:10.1016/j.jad.2011.10.002 
31. Yaghmaie P, Koudelka CW, Simpson EL. Mental health comorbidity in patients with atopic dermatitis. $J$ Allergy Clin Immunol. 2013;131 (2):428-433. doi:10.1016/j.jaci.2012.10.041

32. Nanda MK, LeMasters GK, Levin L, et al. Allergic Diseases and Internalizing Behaviors in Early Childhood. Pediatrics. 2016;137:1. doi: $10.1542 /$ peds. $2015-1922$

33. Toth C, Au S. A prospective identification of neuropathic pain in specific chronic polyneuropathy syndromes and response to pharmacological therapy. Pain. 2008;138(3):657-666. doi:10.1016/j. pain.2008.04.023
34. Li L, Chen J, Wang J, Cai D. Prevalence and risk factors of diabetic peripheral neuropathy in Type 2 diabetes mellitus patients with overweight/obese in Guangdong province, China. Prim Care Diabetes. 2015;9(3):191-195. doi:10.1016/j.pcd.2014.07.006

35. De Vito P, Incerpi S, Pedersen JZ, Luly P, Davis FB, Davis PJ. Thyroid hormones as modulators of immune activities at the cellular level. Thyroid. 2011;21(8):879-890. doi:10.1089/thy.2010.0429

36. De Leo S, Lee SY, Braverman LE. Hyperthyroidism. Lancet. 2016;388(10047):906-918. doi:10.1016/s0140-6736(16)00278-6

\section{Publish your work in this journal}

Neuropsychiatric Disease and Treatment is an international, peerreviewed journal of clinical therapeutics and pharmacology focusing on concise rapid reporting of clinical or pre-clinical studies on a range of neuropsychiatric and neurological disorders. This journal is indexed on PubMed Central, the 'PsycINFO' database and CAS, and is the official journal of The International Neuropsychiatric Association (INA). The manuscript management system is completely online and includes a very quick and fair peer-review system, which is all easy to use. Visit http://www.dovepress.com/testimonials.php to read real quotes from published authors. 